\title{
Critical Care Nurses' Experiences of End-of-Life Care: A Qualitative Study
}

\author{
Reni Sulung Utami ${ }^{1}$, Ahmat Pujianto ${ }^{2}$, Dody Setyawan ${ }^{1}$, Elsa Naviati ${ }^{1}$, Nana Rochana ${ }^{1}$ \\ ${ }^{1}$ Department of Nursing, Faculty of Medicine, Universitas Diponegoro, Semarang, Indonesia, \\ ${ }^{2}$ Department of Nursing, Faculty of Health Sciences, Universitas Borneo Tarakan, Indonesia \\ Corresponding Author: Reni Sulung Utami (reni.sulung@fk.undip.ac.id)
}

Received: 30 June 2020

Revised: 7 November 2020

Accepted: 1 December 2020

\begin{abstract}
Background: Patients admitted to the intensive care unit (ICU) may face terminal illness situations, which may lead to death. In this case, the role of critical care nurses shifts from life-sustaining to end-of-life care (EOLC). Nurses' involvement in EOLC varies between countries, even in one country due to differences in religion, culture, organization, laws, cases and patient quality. In Indonesia, research on EOLC in ICU has not been carried out.

Purpose: This study aimed to explore the experiences of critical care nurses in providing EOLC.

Methods: A qualitative study with a phenomenological approach was conducted. Ten critical care nurses having the experiences of caring for dying patients were recruited through a purposive sampling technique for in-depth interviews. Manual content analysis was used to identify themes.

Results: The results of the study found five themes, including the challenge of communication with the family, support for the family, support for the patient, discussion and decision making, and nurses' emotions.

Conclusion: Most of EOLC provided by critical care nurses was focused on the family. They had some challenges in communication and decision making. Nurses need to get training and education about how to care for patients towards the end of life.
\end{abstract}

Keywords: Critical care nurse; end of life care; experience; intensive care unit

How to Cite: Utami, R. S., Pujianto, A., Setyawan, D., Naviati, E., \& Rochana, N. (2020). Critical care nurses' experiences of end-of-life care: A qualitative study. Nurse Media Journal of Nursing, 10(3), 260-274. doi:10.14710/nmjn.v10i3.31302

Permalink/DOI: https://doi.org/10.14710/nmjn.v10i3.31302

\section{BACKGROUND}

Death is a common occurrence in the intensive care unit (ICU) due to the patient's poor condition. Traditionally, the mortality rate of critically ill patients in the ICU is the highest compared to the mortality rate in any other wards of a hospital. A study showed the average ICU mortality rates worldwide were $16.2 \%$ (Vincent et al., 2014). These deaths occur either anticipated and unexpected. In this situation, the role of critical care nurse shifts from providing actions to maintain life to end-of-life care (EOLC) (Espinosa, 
Young, Symes, Haile, \& Walsh, 2010). Thus, knowledge of end-of-life care is highly recommended for health care providers in ICU.

According to World Health Organization (WHO), EOLC aims to improve the quality of life of patients and families by helping them to overcome various physical, psychological, social and spiritual problems. Implementation of quality EOLC in the intensive care unit (ICU) can be very challenging considering the focus of service in the ICU is to maintain life (Griffiths, 2019). The other studies also address some challenges in implementing EOLC in the ICU, such as inadequate experience and knowledge to provide EOLC, situational conflict to determine the best treatment of EOLC (Kim et al., 2019), difficulties in communication and decision making, and unrealistic family expectation (Brooks, Manias, \& Nicholson, 2017).

The practice of EOLC in the ICU varies significantly between countries. Variability is also found within countries and between intensivists within hospitals. Differences in religion, law, culture, organization, severity of illness, and complexity of cases, as well as the patient's prognosis and quality of life, are believed to play a role in creating this variability (DeCato et al., 2013; Muni, Engelberg, Treece, Dotolo, \& Randall, 2011; Ranse, Yates, \& Coyer, 2016a). It is supported by Kim et al. (2019)'s study claiming that hospital policy and environmental constraints have become some hindrances in the practice of EOLC in Korea. Meanwhile, Brooks et al. (2017) point out that culture, collaboration, and communication are important to yield safe and high quality EOLC in Australia. The latter researchers also recommend to develop EOL care leader and patient support coordinators with nursing and/or medical background.

Nurses have an important position in providing quality EOLC (Zomorodi \& Lynn, 2010). Nurses can be a resource and support for patients and families. Nurses provide guidance for them confronting difficult decisions and adapting to painful realities. King and Thomas (2013) state that nurses accept the reality of death and show a strong commitment to making death as comfortable as possible, more peaceful and dignified. However, providing the best care at the end-of-life is a challenge for them (Coombs, AddingtonHall, \& Long-Sutehall, 2012; Griffiths, 2019). EOLC processes expose nurses to human suffering and miserable situations, thus creating anxiety and uncertainty (Peters et al., 2013) as well as physical and emotional exhaustion (Ozga, Woźniak, \& Gurowiec, 2020; Ryan \& Seymour, 2013).

Nurses' education and experiences related to EOLC will have an impact on the application of end-of-life practices. A study mentioned the importance of nurses' experiences in gaining knowledge to handle end-of-life situations (Ranse, Yates, \& Coyer, 2016b). This knowledge helps them in professional activities for handling difficult situations when caring for dying patients. However, lacking of experience and knowledge of nurses in dealing with EOLC results in negative expressed feelings like sorrow, guilt, regret, and even numbness (Kim et al., 2019).

Research on EOLC has been carried out in many countries and has been identified as a research priority in an international Delphi study (Blackwood, Albarran, \& Latour, 2011). However, up to now, this topic is still rarely carried out in Indonesia. Several studies have 
been done (Fitriyani, Juniarto, \& Utami, 2018; Rochmawati, Wiechula, \& Cameron, 2018; Wessner, 2018), yet the focus is more on the family and religion in palliative care. Only one study was found to explore nurse's experience in carrying out EOLC in West Java (Safitri, Trisyani, \& Iskandar, 2017) though its results remain inadequate to describe Indonesian critical nurses' experiences providing EOLC within broad literature. Enriching literature to get comprehensive understanding of Indonesian critical nurses' experience related to EOLC is necessarily needed by conducting more studies in this area. Therefore, studies on different cultures within Indonesian context should be done.

\section{PURPOSE}

This study aimed to explore the experiences of critical care nurses in providing EOLC.

\section{METHODS}

\section{Research design and setting}

The present research was a qualitative study with a phenomenological descriptive design. Phenomenology was considered ideal for this study because it enables researchers to understand the lived experiences of the human being in the context of their world (Polit \& Beck, 2017). The phenomenon explored in this study was the critical nurses' lived experience in performing EOLC. This research was carried out in the ICU and Pediatric Intensive Care Unit (PICU) of two public hospitals in the province of Central Java, Indonesia, at the end of 2018. In order to uncover the phenomenon, after the research design was conveyed and the research setting was described, the researchers then conducted some systematic steps, such as participant recruitment, data collection and their storage, data analysis, and ensuring trustworthiness (Polit \& Beck, 2017).

\section{Participants and sampling}

A purposive sampling technique was used to recruit ten nurses from ICU/ PICU who met the inclusion and exclusion criteria. Criteria for inclusion of participants in this study were nurses working in ICU/PICU for at least one year, permanent employees and had experiences in caring for patients with end-of-life conditions. The recruitment of participants was done after ethical clearance and research permits from two hospitals were obtained. The researchers approached and delivered information to the head nurses at the research location about the objectives and the research procedures. The researchers asked them for help to obtain data on nurses in their units to identify potential participants. Together with the head nurses, the researchers identified potential nurses as participants based on established inclusion and exclusion criteria. Involving the head nurses was done as they in nature had a good view and consideration about the duty and working experience of their nurses according to the inclusion and exclusion criteria. After obtaining the names of the potential participants and their contact numbers, only ten participants from the list were approached and contacted for further interviews. This process did not involve the head nurses to maintain participants' privacy.

\section{Data collection}

In-depth interviews using semi-structured question sheets were used as data collection methods in this study. The interview questions were developed from the literature review 
and consulted with clinical experts. Participants were invited to take part in interviews by phone. After they agreed to participate in the interview, both parties made a contract on where and when the interviews were carried out at the participant's convenience. This semi-structured interview was conducted for approximately 40-50 minutes in a quiet, comfortable, and closed room and was recorded using a digital recorder. Each participant was interviewed more than once. The interview began with the introduction, signing informed consents, and some general questions. Some open-ended questions were then asked to gain their experiences, like "Could you tell me your experience in looking after the end-of-life patients and their families in this unit?" and "What do you feel when you deal with the patients in their end-of-life and their families?". Some probing questions were asked to clarify or to seek more information about participants' responses, such as "What do you mean by difficulty here?" and "Could you tell me more about this emotion?". Observational field notes/ memos were written throughout the interview process. After all questions were asked, the researchers thanked the participants and asked for their oral consent for a follow-up interview. Each interview record was then labeled in the form of a participant identification number, interview time, and sequence of interviews and field notes during the interview. Furthermore, the recording was transferred to three duplicate audio files stored on a computer, external hard disk (flash disk) and CD before being transcribed verbatim. The stored files on the computer and flash disk were locked for confidentiality and security and only the reseachers knew the password. The interview and participant recruitment were stopped after 10 interviews to the point of data saturation.

\section{Data analysis}

Data collected in this study were analyzed using manual content analysis which was divided into four stages (Polit \& Beck, 2017). The first stage was the process of verbatim recording transcripts and the elimination of the participant's self-identity. The second stage was the process of reading transcripts accompanied by listening to recordings repeatedly to get general conclusions. This process was important to get holistic sense (the gestalt) and overall understanding of the participant's information. The third stage was the process of identifying keywords to produce categories before combining them into sub-themes and themes and looking for relationships between data using maps and diagrams. The fourth stage was the process of integration and interpretation of themes and sub-themes into meaningful descriptions of the phenomena under study.

\section{Data quality}

Ensuring validity and trustworthiness was maintained throughout this study. Validity in term of maintaining the objectivity of the researchers was done through bracketing from the literature review process to the data analysis. Bracketing prevented the researchers' personal views and preconceptions from interfering the unique descriptions of the phenomenon. It also prevented the researchers' interpretation during data analysis. Prior knowledge and interpretation were bracketed and written down on memos. Furthermore, the trustworthiness of this study was ensured by conducting triangulation with the theory and secondary data (documents, field notes), member checking, peer debriefing, and audit trail. Member checking was carried out by having follow-up interviews for clarification and further discussion. However, only four out of ten participants had done the member checking due to time constraints. Peer debriefing was done through researcher team's 
meetings to review and discuss the transcripts, keywords, categories, and themes while audit trail was conducted by collecting and documenting the data systematically so that the data could be reviewed externally.

\section{Ethical consideration}

This research had obtained an ethical clearance prior to the data collection from the Health Research Ethics Committee (KEPK) of the Faculty of Medicine, Universitas Diponegoro (No. 250/EC/FK_RSDK/IV/2018), and permissions from two hospitals where this study was taken place. A statement letter explaining the research and an informed consent letter as a participant was given to participants before interviews to respect the dignity and the freedom of the participants and to avoid being coerced into participating in this research. Participants who agreed to be involved in the study were asked to sign an informed consent form. Privacy and confidentiality of participants were also maintained in this study. The use of pseudonyms to identify the participants was done to keep the confidentiality.

\section{RESULTS}

\section{Characteristics of participants}

As presented in Table 1, this study involved ten nurses working in the ICU/PICU. A majority of them were females, aged 41 to 50 years old, graduated from bachelor and nursing professional degree and had working experiences of two to ten years.

\section{Table 1 Characteristics of participants}

\begin{tabular}{ll}
\hline Characteristics & $f(\%)$ \\
\hline Gender & $2(20)$ \\
Male & $8(80)$ \\
Female & \\
\hline Age (years) & $1(10)$ \\
$21-30$ & $3(30)$ \\
$31-40$ & $6(60)$ \\
$41-50$ & \\
\hline ICU experience (years) & $6(60)$ \\
$2-10$ & $2(20)$ \\
$11-20$ & $2(20)$ \\
$>20$ & \\
Level of education & $4(40)$ \\
DIII & $1(10)$ \\
DIV & $5(50)$ \\
S1 + Ners & \\
\hline
\end{tabular}

The results of the thematic analysis of nurses' experiences in providing EOLC to patients in the ICU revealed five main themes namely the challenges of communication with family, support for the family, support for the patients, discussion and decision making, and nurses' emotions. Each of these themes is presented and illustrated using verbatim quotes from interviews. 


\section{Theme 1: The challenges of communication with the family}

Participants stated that communicating with families with end-of-life conditions has its own challenges and difficulties. The communication difficulties faced by nurses primarily are communicating with families who have high expectations, families are still in the denial stage, and smart-assed families. Higher and lower education does not always guarantee the ease of communication. The participants' statements are quoted below:

"The challenge is the family, ... sometimes the family has been informed, but the family has not been able to accept the patient's condition ..." (P7)

"I thought that communicating with the family with higher education will be easy; but it is not; they even need more details... For family who had low education..., giving knowledge is also difficult. It turns out that education also determines the standpoint of the other side... So, the family's response must be meticulous too. It was also difficult to provide education to people who work in health field as similar with us..." (P6)

"... The family still hopes that the patient is be able to return; it is difficult to tell the family that this is hopeless, and they also have difficulty in understanding the medical terms..." (P9)

"It's difficult to handle family who acts know it all...; family with a high-income and higher education... They can understand when they are given explanation, but there are also some families who are pretentious to understand... They did not fully understand... However, there are also some families that are highly educated but it is difficult to talk to them. It's just difficult to explain to them..." (P2)

One participant said that nurses' ability to communicate significantly determines the relationship of nurses, both with colleagues and family. Level of education of family affects the communication techniques that nurses applied.

"...Our ability to communicate determines both partners and family... The level of education of family varies, so the language we choose determines whether or not he can accept it. We have to adjust to their level of education so that they can understand if we use a common imagery. Maybe our techniques for conveying need different ways..." (P10)

The existence of a case conference that decides an agreement between the health team regarding what the family will convey is perceived to be very helpful for the participants in communicating with the family. This was stated by the participants as below:

"So, nurses and physicians convey their feelings and voices related to patient care in the case conference and then a decision will be made... We talk to the family based on the script that we agreed upon, for example A to A or B to B. This conference is very helpful for us..." (P2)

\section{Theme 2: Support for families}

This theme emphasizes the form of support provided by nurses to families of patients with end of life care plans. This support included giving encouragement, trying to always 
be there for the family, and inviting families to chat and pray together. This was stated by the participants as below:

"So, we can only give encouragement to his family..., and providing understanding to his family in order to accept the reality... The most important thing for me to support him is being close to him, accompanying him, and inviting him to discuss about his son problems so he can accept any circumstances related to his son... Basically, communication is the key to being close with him... Do not leave him alone...; invite him to prayer together as well..." (P8)

"...we give understanding that the patient cannot be helped and we have tried to help patient optimally..., but the patient did not want to be helped anymore... We explain it to both parents even though it was in sad situation... At least, we encourage them to talk to reduce their grief rather than only said to them that their child is dead and we are busy taking care the deceased... It looks like we left them alone... If we ask them to talk, they will not feel sad alone." (P9)

Another form of support provided by nurses is to provide opportunities for families to always be close to patients. Families are allowed to be near the patient even outside the visiting hours, but this applies only to the nuclear family, for example the patient's parents.

"... two family members are allowed to wait in the bedside of a dying patient... We gave them the opportunity even though it was out of visiting hours..." (P3)

"...those conditions sometimes trigger our social spirit. We allow the caregiver sit near the patient's bed even though it was not the visiting hours. All curtains were closed. We also often accompany them so that patients can be accompanied continuously." (P5)

"He was allowed to pray together with his family's member or his religious leader... He is permitted to approach his child to pray until his child death." (P8)

\section{Theme 3: Support to patients}

Support is given to patients as an effort to raise comfort to patients such as avoiding actions that cause pain, providing pain medication and providing personal hygiene care. Almost all participants gave spiritual support to patients, either directly or indirectly, for example, listening to spiritual texts (e.g., the Holy Quran recitation), guiding prayers, and facilitating spiritual guidance by calling religious leader as needed.

“...Improve the quality of life of patients by providing a sense of comfort, for example by reducing pain ..." (P7)

"... if it has been stated end-of-life, we are more focus on patient comfort, for example, performing personal hygiene, positioning, and persuading the physician to avoid taking unnecessary blood samples... That's the focus of nursing care..." (P9)

"...we give analgesic to make the patient feel comfortable, perform maximal wound care, nutrition is also maximized. We support family in order to continue in providing support for patient" (P6) 
"For Moslem patients, usually while bathing, they recite Al Fatihah or healing prayers. If the patients are still conscious, we encourage them to recite Al Fatihah... When I give injection, I ask them to pray... Hopefully later, through this drug, the God can give the cure" (P2)

"For spiritual care, it's rather difficult if the patient is not fully conscious...; at least by playing Al Quran recitation.” (P6)

"...for patients and families... so we offer it to the family ... do they need spiritual support/guidance?... in our hospital there are Islamic and Christian spiritual facilities ... if necessary we will facilitate ..." (P1)

\section{Theme 4: Discussion and decision making}

Majority of participants stated that decisions related to care provided to patients were made based on the results of the discussion between family and the multidisciplinary team; yet the role of physicians is dominant compared to that of nurses. Nurses tend to follow the doctor's instructions.

"...for cases of long-stay patient, we usually have discussions with family and team such as anesthetist, neurologist, pulmonologist, and others. We sit and discuss together then we made a decision..." (P5)

"...if patients stay for more than 10 days or more than 2 weeks... We always carry out case conferences... the result will be informed to the family for further followup... for long stay patients or patients with complicated diagnosis." (P2)

"The nurse is the first one who knows for sure... We share it first to the doctor... later the doctor will educate the family... Even though we can do it, but we always share with the doctor... We didn't make decisions... It is because in the informed consent, the doctor is the one who gives the signature... but the doctor knew the issue from the nurse..." (P2)

"If the patient has been declared dying, we will wait until the time is up... then what the advice of the doctor? If for example, he has bradycardia/ asystole...., there is no hope anymore and then the doctor says it is finished... Yeah it means finished." (P8)

However, there were some participants who have a courage to argue with doctors regarding patient care. That happen if they had a strong justification.

"...actually, I disagree, but this is a team... So I give help too... We had an argument... our last strategy was calling the family..." (P9)

“... actually, it doesn't make any difference, even though it is end of life... We still support the respiration, their nutrition and others..., but these supports depend on the physician... but we can propose, for example giving analgesia... We can propose it to the doctor because it's his area, not ours. But still we can propose it with a note if there is a reason." (P10)

\section{Theme 5: Nurses' emotions}

Participants said that caring patients of end-of-life reminded them of death. Participants conveyed sympathy and empathy to both patients and families. Participants could feel the sorrow of the family who lost their member and even cried. 
“... if saw the patient is still productive and the disease is not too complicated, there is such a pity feeling... Why not helping up to the maximum first... Once there was a family that is given up; it might be because of the cost or something. We always explain that this patient can be improved... why did not withhold?... But, if the patient is on end stage...for example cancer stage 4, elderly with stroke..., we just remember about dies; we will also experience death someday...." (P2)

"The most imprint is when they have a son...; to have a son is already difficult or has many times but miscarriage, and this is the only child he has... It continues to imprint in our heart...feel a pity... extraordinary empathy... Because we are also a human and a woman can feel how she has a difficulty in having child but God does not grant it" (P8)

"... sometimes, when the family has been told that their child is dying... I am emotionally crying too..." (P10)

One participant said the death rate in her ward was quite high and this caused fears on her that it will reduce her empathy due to seeing patients die so often.

“... I am afraid that my heart will harden... I am afraid that I am less sensitive to people's sadness because I often see people die... When the patient dies, the family cries and we can still laugh..." (P4)

\section{DISCUSSION}

This study aimed to explore the nurses' experiences in providing EOLC in the ICU. The findings showed that nurses had several challenges in providing the care. Moreover, most of EOLC provided by nurses was focused on family. Some studies state that EOLC in the ICU is challenging (Kisorio \& Langley, 2016; Ozga et al., 2020). Ozga et al (2020) identified three categories of potential barriers in providing EOLC, namely (1) barriers attributable to the hospital, (2) barriers related to the patient's family, and (3) barriers related to the ICU personnel providing direct EOLC.

In this study, participants reported some challenges and difficulties when communicating with the family of dying patients. These results are consistent with the results of previous studies (Aslakson et al., 2012; Crump, Schaffer, \& Schulte, 2010; Muni et al., 2011; Ozga et al., 2020). There are several factors that can create challenges and have an impact on the quality and quantity of communication between doctors, patients, and families at the end-of-life in the ICU. These factors include communication skills of doctors and nurses, lack of time of doctors/nurses, the ability of families to understand the information delivered, cultural disparities and the emotional condition of the family (Aslakson et al., 2012; Jin, 2013; Levin, Moreno, Silvester, \& Kissane, 2010). All these factors were stated by participants in this study. Nurses in this study convey difficulties when communicating with families who are still in the denial phase. Denial is a common coping mechanism for those facing a terminal illness. In this stage, families start to deny bad news that is given. They believed that the information is wrong and there must have been a mistake. In dealing with this situation, nurses must respect the family's essentially protective nature of it. Nurses need to seek first to understand and validate before attempting to change anything. This will produce far more therapeutic benefits than trying to change or override such defenses. Encouraging positive aspects of the family and building them as strengths 
may be more beneficial than confrontation and criticism. Nurses need to master these skills.

The complexity of information, family's lack of capacity in understanding information, and the uncertainty of critical patient conditions can make family members feel depressed and frustrated (Carlson, Spain, Muhtadie, McDade-Montez, \& Macia, 2015). This emotional disturbance can interfere with cognitive processes and affect the ability of families to understand, remember, and search for complex information about the condition and prognosis of their loved ones (Morgado \& Cerqueira, 2018; Sandi, 2013). In 2015, Mathew, Azariah, George, and Grewal reported that $71 \%$ of families have a poor understanding of the diagnosis, treatment, and prognosis of patients. Family's understanding will get worse when the family only interacts with the healthcare staff for less than 10 minutes.

Nurses spent the most amount of their time with patients and families compared to other health care professionals; thus, it gave nurses a great opportunity to build trust and give comfort care. Nevertheless, they still feel they are not sufficiently prepared to care for dying patients and their families (Espinosa et al., 2010). Nurses who are inexperienced and not ready to provide care can experience doubts about what should be done and, in the end, do nothing (Kisorio \& Langley, 2016). In this study, the inexperienced nurses sought for help from their senior experienced nurses.

Families feel the presence of nurses as comforting hugs that sustain them before, during and after the death of the patient (Williams, Lewis, Burgio, \& Goode, 2012). In this study, some participants provide time for families to accompany them and listen to their feelings and thoughts even though it was not always possible. Being present allow nurses to experience the families' lives, sensing their milieu and their situation. As a result, it could enhance their empathy and compassionate behavior to others. Indeed, for some nurses, this practice is not easy task for them because they do not feel confident from their lack of knowledge and experience. In Indonesia, training and nursing curriculum related to end of life care is likely to be inadequate. This issue also found in other settings (Cavaye \& Watts, 2010).

To ensure patient comfort in the dying process, nurses provide spiritual care and basic nursing care. In this study, nurses encouraged and guided patients and their families to pray and facilitated them in inviting a religious leader. Nurses also allowed them to bring their own spiritual leader. Spiritual care plays a vital role in preparing for a peaceful death and is an important aspect of holistic nursing care (Witt Sherman \& Free, 2015). Spiritual care is not only important for family, but also for family member and medical staff. The positive effects of spiritual care have been reported (Chen, Lin, Yan, Wu, \& Hu, 2018; Willemse et al., 2020; Zhang, Nilsson, \& Prigerson, 2013). A recent ethnographic study in Indonesia found that spirituality/religiosity is very important in the daily lives of patients, family members and healthcare staff (Rochmawati et al., 2018). Even though spiritual care is viewed as an important dimension of end-of-life care, its implementation is still inadequate (Balboni et al., 2013). This phenomenon may be caused by the lack of training and education. 
End-of-life decision making is a growing need in the ICU, covering a continuum of treatment possibilities. It has been perceived as complex, difficult and stressful (Pereira, Fradique, \& Hernandez-Marrero, 2018). This study reveals that families are involved in the decision-making process. In Indonesian culture context, the role of family is strong and important in patient care. Moreover, discussion involving family is essential because patient in high vulnerability condition and generally unconscious.

In line with previous research (Flannery, Peters, \& Ramjan, 2020; Griffiths, 2019; Kisorio \& Langley, 2016), the role of nurses in making decisions regarding the end-of-life in this study is still lacking. They still have a tendency to follow doctor's instructions. This could happen because end-of-life decision is a medical decision and becomes the responsibility of physicians. On the other hand, nurses often feel inadequately prepared (Griffiths, 2019). Interprofessional education and collaboration between physician and nurse can improve future decision-making processes (Flannery et al., 2020).

Nurses are frequently exposed to the care of death and dying patients. This experience made them aware of their own death (Peters et al., 2013). This is as stated by participants in this study. Peters et al (2013) reveals that exposure to death often causes anxiety in nurses. Senior nurses with more experiences tended to have more positive attitudes toward death and caring for dying patients, whereas nurses without this experiences had more negative attitudes (Abu Hasheesh, Abozeid, El-Said, \& Alhujaili, 2013). Hinderer (2012) stated that understanding nurses' emotions to patient death can help to improve the quality of care they provided to dying patients and to meet the needs of the nurses who care for them.

The reactions of participants in this study conveyed sympathy and empathy to both patient and family. Sympathy was described as a superficial acknowledgment of suffering, generating a compassionate response that fails to adequately acknowledge the person who is suffering. In contrast, empathy is an affective response that recognizes and tries to understand individual suffering through emotional resonance (Sinclair et al., 2017). Empathy has a positive meaning and more welcomed and valued by patient and families.

This study reveals that caring for the dying patients was challenging emotionally and psychologically for nurses. These challenges must be addressed. Nurses need to enhance their communication skills and more actively involved in facilitating the end-of-life decision making process. Education and training about EOLC are imperative for providing the best quality of EOLC. In addition, a course in palliative care is suggested to be mandatory in nursing education.

A few limitations of this study should be acknowledged. Some participants were interviewed at the ICU because they could not leave the ward and refused to meet after working hours. As a result, the interview process is sometimes interrupted by the activities of other nurses in the room. Member checking was not carried out to all participants due to time constraints resulting in possible researchers' bias though bracketing and peer debriefing had been done.

\section{CONCLUSION}


This study showed five themes, namely the challenges of communication with family, support to the family, support to the patients, discussion and decision making, and nurses' emotions. Nurses had several challenges and difficulties in communicating with the family of dying patients. Moreover, EOLC provided by nurses was more focused on family involvement. Nurses were still not optimal in providing care for patients in endof- life condition. Nurses need to get training and education about how to care for patients towards the end-of-life. Further research needs to be carried out to explore nurses' communication experiences related to EOLC.

\section{ACKNOWLEDGMENT}

We gratefully thank the Faculty of Medicine, Universitas Diponegoro for financial support.

\section{CONFLICT OF INTEREST}

The authors declare that they have no conflict of interests

\section{REFERENCES}

Abu Hasheesh, M. O., Abozeid, S. A. S., El-Said, S. G., \& Alhujaili, A. D. (2013). Nurses' characteristics and their attitudes toward death and caring for dying patients in a public hospital in Jordan. Health Science Journal, 7(4), 384-394.

Aslakson, R. A., Wyskiel, R., Thornton, I., Copley, C., Shaffer, D., Zyra, M., ..., \& Pronovost, P. J. (2012). Nurse-perceived barriers to effective communication regarding prognosis and optimal end-of-life care for surgical ICU patients: A qualitative exploration. Journal of Palliative Medicine, 15(8), 910-915. doi:10.1089/jpm.2011.0481

Balboni, M. J., Sullivan, A., Amobi, A., Phelps, A. C., Gorman, D. P., Zollfrank, A., ..., \& Balboni, T. A. (2013). Why is spiritual care infrequent at the end of life? spiritual care perceptions among patients, nurses, and physicians and the role of training. Journal of Clinical Oncology, 31(4), 461-467. doi:10.1200/JCO.2012.44.6443

Blackwood, B., Albarran, J. W., \& Latour, J. M. (2011). Research priorities of adult intensive care nurses in 20 European countries: A Delphi study. Journal of Advanced Nursing, 67(3), 550-562. doi:10.1111/j.1365-2648.2010.05512.x

Brooks, L.A, Manias, E., \& Nicholson, P. (2017). Barriers, enablers and challenges to initiating end-of-life care in an Australian intensive care unit context. Australian Critical Care, 30, 161-166. doi:10.1016/j.aucc.2016.08.001

Carlson, E. B., Spain, D. A., Muhtadie, L., McDade-Montez, L., \& Macia, K. S. (2015). Care and caring in the intensive care unit: Family members' distress and perceptions about staff skills, communication, and emotional support. Journal of Critical Care, 30(3), 557-561. doi:10.1016/j.jcrc.2015.01.012

Cavaye, J., \& Watts, J. H. (2010). End-of-life education in the pre-registration nursing curriculum: Patient, carer, nurse and student perspectives. Journal of Research in Nursing, 17(4), 317-326. doi:10.1177/1744987110379531

Chen, J., Lin, Y., Yan, J., Wu, Y., \& Hu, R. (2018). The effects of spiritual care on quality of life and spiritual well-being among patients with terminal illness: A systematic review. Palliative Medicine, 32(7), 1167-1179. doi:10.1177/0269216318772267

Coombs, M., Addington-Hall, J., \& Long-Sutehall, T. (2012). Challenges in transition from intervention to end of life care in intensive care: A qualitative study. 
International Journal of Nursing Studies, 49(5), 519-527. doi:10.1016/j.ijnurstu.2011.10.019.

Crump, S. K., Schaffer, M. A., \& Schulte, E. (2010). Critical care nurses' perceptions of obstacles, supports, and knowledge needed in providing quality end-of-life care. Dimensions of Critical Care Nursing, 29(6), 297-306. doi:10.1097/DCC.0b013e3181f0c43c

DeCato, T. W., Engelberg, R. A., Downey, L., Nielsen, E. L., Treece, P. D., Back, A. L., ..., \& Curtis, J. R. (2013). Hospital variation and temporal trends in palliative and end-of-life care in the ICU. Critical Care Medicine, 41(6), 1405-1411. doi:10.1097/CCM.0b013e318287f289

Espinosa, L., Young, A., Symes, L., Haile, B., \& Walsh, T. (2010). ICU nurses' experiences in providing terminal care. Critical Care Nurse, 33(3), 273-281.

Fitriyani, N., Juniarto, A. Z., \& Utami, R. S. (2018). Life quality of end of life patient's family in intensive care units. Indonesian Journal of Nursing and Midwifery, 6(1), 16-24. Retrieved from http://ejournal.almaata.ac.id/index.php/JNKI

Flannery, L., Peters, K., \& Ramjan, L. M. (2020). The differing perspectives of doctors and nurses in end-of-life decisions in the intensive care unit: A qualitative study. Australian Critical Care, 33(4), 311-316. doi:10.1016/j.aucc.2019.08.004

Griffiths, I. (2019). What are the challenges for nurses when providing end-of-life care in intensive care units? British Journal of Nursing, 28(16), 1047-1052.

Hinderer, K. A. (2012). Reactions to patient death: The lived experience of critical care nurses. Dimensions of Critical Care Nursing, 31(4), 252-259. doi:10.1097/DCC.0b013e318256eff1

Jin, J. (2013). Clinicians examine advances and challenges in improving quality of endof-life care in the ICU. JAMA, 310(23), 2493-2495. doi:10.1001/jama.2013.283830

Kim, S., Savage, T. A., Song, M. K., Vincent, C., Park, C. G., Ferrans, C. E., \& Cavanaugh, K. (2019). Nurses' roles and challenges in providing end-of-life care in neonatal intensive care units in South Korea. Applied Nursing Research, 50, 151204. doi:10.1016/j.apnr.2019.151204

King, A., \& Thomas, P. (2013). Phenomenological study of ICU nurses'experiences caring for dying patients. Western Journal of Nursing Research, 35(10), 1292-1308.

Kisorio, L. C., \& Langley, G. C. (2016). Intensive care nurses' experiences of end-of-life care. Intensive and Critical Care Nursing, 33, 30-38. doi:10.1016/j.iccn.2015.11.002

Levin, T. T., Moreno, B., Silvester, W., \& Kissane, D. W. (2010). End-of-life communication in the intensive care unit. General Hospital Psychiatry, 32(4), 433442. doi:10.1016/j.genhosppsych.2010.04.007

Mathew, J. E., Azariah, J., George, S. E., \& Grewal, S. S. (2015). Do they hear what we speak? Assessing the effectiveness of communication to families of critically ill neurosurgical patients. Journal of Anaesthesiology, Clinical Pharmacology, 31(1), 49-53. doi:10.4103/0970-9185.150540

Morgado, P., \& Cerqueira, J. J. (2018). Editorial: The impact of stress on cognition and motivation. Frontiers in Behavioral Neuroscience, 12, 326. doi:10.3389/fnbeh.2018.00326

Muni, S., Engelberg, R. A., Treece, P. D., Dotolo, D., \& Randall, J. (2011). The influence of race / ethnicity and socioeconomic status on end-of-life care in the intensive care unit. CHEST Journal, 139(5), 1025-1033. doi:10.1378/chest.10-3011 
Ozga, D., Woźniak, K., \& Gurowiec, P. J. (2020). Difficulties perceived by ICU nurses providing end-of-life care: A Qualitative Study. Global Advances in Health and Medicine, 9, 1-8. doi:10.1177/2164956120916176

Pereira, S. M., Fradique, E., \& Hernandez-Marrero, P. (2018). End-of-life decision making in palliative care and recommendations of the Council of Europe: Qualitative secondary analysis of interviews and observation field notes. Journal of Palliative Medicine, 21(5), 604-615. doi:10.1089/jpm.2017.0403

Peters, L., Cant, R., Payne, S., O’Connor, M., McDermott, F., \& Hood, K. (2013). How death anxiety impacts nurses' caring for patients at the endof life: A review of literature. The Open Nursing Journal, 7, 14-21.

Polit, D. F., \& Beck, C. T. (2017). Nursing research: Generating and assessing evidence for nursing practice (Tenth). Philadelphia: Wolters Kluwer Health.

Ranse, K., Yates, P., \& Coyer, F. (2016a). End-of-life care practices of critical care nurses: A national cross-sectional survey. Australian Critical Care, 29(2), 83-89. doi:10.1016/j.aucc.2015.07.001

Ranse, K., Yates, P., \& Coyer, F. (2016b). Modelling end-of-life care practices: Factors associated with critical care nurse engagement in care provision. Intensive \& Critical Care Nursing: The Official Journal of the British Association of Critical Care Nurses. doi:10.1016/j.iccn.2015.11.003

Rochmawati, E., Wiechula, R., \& Cameron, K. (2018). Centrality of spirituality/religion in the culture of palliative care service in Indonesia: An ethnographic study. Nursing and Health Sciences, 20(2), 231-237. doi:10.1111/nhs.12407

Ryan, L., \& Seymour, J. (2013). Death and dying in intensive care: Emotional labour of nurses. End of Life Journal, 3(2), 1-9.

Safitri, A., Trisyani, Y., \& Iskandar, A. A. (2017). An exploration of nurse experience in caring for end of life care client and family in critical care setting in Indonesian context. Journal of Advances in Health and Medical Sciences, 3(2), 53-62. doi:10.20474/jahms-3.2.2

Sandi, C. (2013). Stress and cognition. Wiley Interdisciplinary Reviews. Cognitive Science, 4(3), 245-261. doi:10.1002/wcs.1222

Sinclair, S., Beamer, K., Hack, T. F., Mcclement, S., Bouchal, S. R., Chochinov, H. M., \& Hagen, N. A. (2017). Sympathy, empathy, and compassion: A grounded theory study of palliative care patients' understandings, experiences, and preferences. Palliative Medicine, 31(5), 437-447. doi:10.1177/0269216316663499

Vincent, J.-L., Marshall, J. C., Ñamendys-Silva, S. A., François, B., Martin-Loeches, I., Lipman, J., ..., \& Sakr, Y. (2014). Assessment of the worldwide burden of critical illness: the Intensive Care Over Nations (ICON) audit. The Lancet Respiratory Medicine, 2(5), 380-386. doi:10.1016/S2213-2600(14)70061-X

Wessner, P. B. (2018). Last chance to care: An autoethnography of end-of-life care in Indonesia. The Qualitative Report, 23(9), 2238-2250.

Willemse, S., Smeets, W., Van Leeuwen, E., Nielen-Rosier, T., Janssen, L., \& Foudraine, N. (2020). Spiritual care in the intensive care unit: An integrative literature research. doi:10.1016/j.jcrc.2020.01.026

Williams, B. R., Lewis, D. R., Burgio, K. L., \& Goode, P. S. (2012). Next-of-Kin's perceptions of how hospital nursing staff support family presence before, during, and after the death of a loved one. Journal of Hospice \& Palliative Nursing, 14(8), 541550. doi:10.1097/NJH.0b013e31825d2af1 
Witt Sherman, D., \& Free, D. C. (2015). Culture and spirituality as domains of quality palliative care. In M. Matzo \& W. S. Deborah (Eds.), Palliative Care Nursing (4th ed., pp. 91-2015). New York: Springer Publishing Company. doi:10.1891/9780826196361.0005

Zhang, B., Nilsson, M. E., \& Prigerson, H. G. (2013). Factors important to patients' quality-of-life at the end-of-life. Arch Intern Med, 172(15), 1133-1142. doi:10.1001/archinternmed.2012.2364.Factors

Zomorodi, M., \& Lynn, M. R. (2010). Critical care nurses' values and behaviors with end-of-life care: Perceptions and challenges. Journal of Hospice \& Palliative Nursing, 12(2), 89-96. doi:10.1097/NJH.0b013e3181cf7cf6 